\title{
A ENFERMAGEM PEDIÁTRICA ANTE AS REPERCUSSÕES DO CUIDAR DA CRIANÇA ONCOLÓGICA: UMA REVISÃO DE LITERATURA
}

\section{PEDIATRIC NURSING IN VIEW OF ONCOLOGIC CHILD CARE REPERCUSSIONS: REVIEW}

\author{
Vanessa do Nascimento Silveira, ${ }^{1}$ Danúbia Moraes Legramante, ${ }^{1}$ Greice Machado Pieszak ${ }^{1}$ \\ ${ }^{1}$ Universidade Integrada do Alto Uruguai e das Missões - URI/Santiago-RS/Brasil. \\ Autor correspondente: Vanessa do Nascimento Silveira \\ e-mail: vanessa.sylveira@yahoo.com.br
}

\section{EDITORES}

Thiago Gomes Heck

(Unijuí-Brasil)

Adriane Cristina Bernat Kolankiewicz

(Unijuí-Brasil)

\section{EDITORES DE ÁREA}

Educação \& Saúde

Eva Teresinha de Oliveira Boff

(Unijuí-Brasil)

Fisioterapia \& Saúde

Eliane Roseli Winkelmann

(Unijuí-Brasil)

Ciências Farmacêuticas \& Saúde

Marilei Uecker Pletsch

(Unijuí-Brasil)

Nutrição \& Saúde

Lígia Beatriz Bento Franz

(Unijuí-Brasil)

Nadia Oliveira

(Unipampa-Brasil)

Ingrid Perry

(UNESC-Brasil)

Enfermagem e suas contribuições para a prática

Adriane Cristina Kolankiewicz

(Unijuí-Brasil)

Crhis de Brum

(UFFS-Brasil)

Neila de Souza

(UFSM-Brasil)

Exercício Físico \& Saúde

Thiago Gomes Heck

(Unijuí-Brasil)

Anderson Zampier Ulbrich

(UFPR)

Editora Unijuí

Universidade Regional do Noroeste do

Estado do Rio Grande do Sul (Unijuí)

\section{RESUMO}

Trata-se de uma pesquisa bibliográfica do tipo narrativa de caráter descritivo-exploratório, com abordagem qualitativa, que tem por objetivo analisar as publicações sobre oncologia pediátrica e as implicações para o cuidado de enfermagem. A busca foi realizada em fevereiro de 2016, na base de dados Literatura Latino-Americana e do Caribe em Ciências da Saúde (Lilacs). Utilizou-se os seguintes descritores: "Enfermagem Pediátrica" e "Enfermagem Oncológica". A partir da análise de dados, emergiram três categorias: a ótica do cuidar da criança oncológica no contexto da família e da equipe de enfermagem; a repercussão dos sentimentos e emoções que permeiam o processo de adoecimento; e a promoção da qualidade de vida da criança oncológica e família. Nesse sentido, conclui-se que a equipe de enfermagem não deve se limitar apenas aos protocolos terapêuticos, mas também incluir ações humanizadas que valorizem a participação constante da família nesse contexto, posto que a cura não deve ser baseada apenas no restabelecimento biológico, mas no bem-estar e qualidade de vida dessas crianças.

Palavras-chave: Enfermagem pediátrica; Enfermagem oncológica, Família.

Submetido em: $30 / 3 / 2016$

Aceito em: 19/10/2016 


\section{Revista}

\section{Contexto}

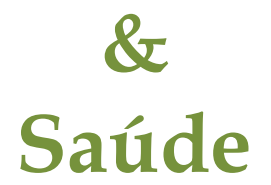

Volume 16

Número 31

2016

ISSN 2176-7114

\begin{abstract}
This is a literature narrative type of descriptive and exploratory nature with a qualitative approach, which aims to analyze the publications on pediatric oncology and the implications for nursing care. The search was conducted in February 2016, the Latin American database and Caribe Health Sciences (Lilacs), we used the following descriptors: "Pediatric Nursing" and "Oncology Nursing". From the data analysis, three categories have emerged: the perspective of caring for the child cancer in the context of family and nursing staff; the impact of the feelings and emotions that permeate the disease process; and the promotion of the family oncological and child quality of life. In this sense, it is concluded that the nursing staff, should not be limited only to therapeutic protocols, but also include humanized actions that value the constant participation of the family in this context, since the cure should not be based solely on biological recovery, but the well-being and quality of life of these children.
\end{abstract}

Keywords: Pediatric nursing. Oncology nursing. Family.

\begin{abstract}
A Revista Contexto \& Saúde é um periódico do Departamento de Ciências da Vida da Universidade Regional do Noroeste do Estado do Rio Grande do Sul (Unijuí). É um periódico semestral que tem por objetivo a divulgação da produção técnico-científica de temas relacionados à área de Ciências da Saúde.

O escopo da revista abrange a divulgação de resultados de pesquisa que contemplem avanços no processo saúde-doençacuidado e no conhecimento e aplicabilidade de novos processos químicos e biológicos em saúde.

Neste periódico, entende-se que a publicação de estudos com os aspectos epidemiológicos, assistenciais e educacionais em saúde, experimentais e aplicados é uma forma a subsidiar e qualificar a atenção à saúde de modo interdisciplinar.
\end{abstract}




\section{INTRODUÇÃO}

O Estatuto da Criança e do Adolescente (ECA) foi instituído pela Lei 8.069, no dia 13 de julho de 1990, para regulamentar os direitos das crianças e dos adolescentes. O mesmo está embasado nas diretrizes da Constituição Federal de 1988. Para o ECA, é considerada criança a pessoa com idade inferior a 12 anos e adolescente aquela entre 12 e 18 anos de idade (BRASIL, 2010).

O processo de adoecimento na criança promove rápida e intensa transformação em sua vida e no cotidiano familiar. A hospitalização inesperada em um ambiente estranho, com pessoas desconhecidas, no qual a criança será submetida a exames e tratamentos invasivos e dolorosos, sendo afastada do seu contexto social, familiares e amigos, que passa a ser o local de maior permanência dela, independentemente da sua idade e capacidade de compreensão cognitiva da realidade que a rodeia, faz, de certa forma, com que ela perceba que algo diferente está acontecendo consigo (GOMES; COLLET; REIS, 2011).

O câncer é um crescimento desordenado de células agressivas e incontroláveis que invadem os tecidos e órgãos e dividem-se rapidamente, determinando a formação de tumores ou neoplasias malignas. No adulto, o câncer afeta as células do epitélio, responsável por recobrir diferentes órgãos, enquanto na criança, geralmente, afeta as células do sistema sanguíneo e os tecidos de sustentação (AVANCI et. al., 2009).

Segundo o Instituto Nacional do Câncer - Inca (BRASIL, 2008), o câncer infantil difere-se do adulto quanto ao local de acometimento primário e principalmente pela sua sintomatologia inespecífica. Às vezes é confundido e tratado equivocadamente como outras doenças comuns para a idade, o que repercute em um diagnóstico tardio.

De acordo com Silva et al. (2013), o câncer infantil compreende um grupo de patologias crônicas não transmissíveis que acomete crianças e adolescentes de zero a 19 anos, com a identificação de células modificadas que se multiplicam rápida e desordenadamente, interferindo no funcionamento de determinado órgão.

Considerado uma doença rara, o câncer infantil representa $1 \%$ a $3 \%$ de todos os tumores malignos das populações. Na maioria das vezes possui curtos períodos de latência, é mais agressivo e cresce rapidamente; responde, no entanto, melhor ao tratamento e é considerado de bom prognóstico. Dentre os tumores mais frequentes na infância e na adolescência, pode-se citar as leucemias, os do sistema nervoso central e os linfomas (BRASIL, 2014).

No Brasil, 2.812 crianças e adolescentes morreram por câncer em 2011. As neoplasias configuram-se como a doença com maior morbimortalidade, pois ocupam a segunda posição (7\%) de óbitos dessa população. Nas estimativas de 2014, o número de casos novos de câncer foi de 394.450, excluindo-se apenas os tumores de pele não melanoma (BRASIL, 2011).

Cabe ressaltar que, atualmente, a cada ano mais de 160.000 crianças são diagnosticadas com câncer e ainda, pesquisas mostram que se estas receberem o prognóstico precocemente possuem $80 \%$ de chance de cura (BRASIL, 2011).

A criança oncológica é considerada um ser que carece de atenção e cuidados especiais em razão de sua ingenuidade perante a realidade. A mesma sofre impactos influenciados por diversos fatores, como a idade e a inexperiência. Sendo assim, a família é o componente essencial na assistência e promoção da saúde desta 
(AVANCI et al., 2009). Diante do exposto, surge a necessidade de o enfermeiro refletir a sua práxis no contexto em que essa criança está inserida, tendo em vista a alta incidência de casos de câncer nos últimos anos no país.

Neste contexto, o cuidado à criança com câncer é complexo, pois envolve múltiplos aspectos, dentre eles culturais, econômicos e psicossociais. Nesse sentido, faz-se necessária a atuação do profissional enfermeiro na oncologia pediátrica, e, além do conhecimento técnico e científico, a afetividade na oferta do cuidado à criança e a sua família com vistas à promoção da saúde, qualidade de vida, conforto e bem-estar dos mesmos (SILVA et al., 2013).

Em razão das internações recorrentes e ao tempo de duração destas, a criança enferma estabelece um vínculo e uma familiaridade com o ambiente hospitalar. Esse processo fortalece a efetivação da assistência de enfermagem a partir da confiança, cuidado e diálogo entre equipe, família e criança. Além disso, surgem emoções e comportamentos correlacionados ao diagnóstico, tratamento e prognóstico da doença, momento em que o enfermeiro deve estar atento para identificar e encontrar a melhor forma de conduzir tais situações (AVANCI et al., 2009).

Nessa perspectiva, cabe ao enfermeiro reconhecer as potencialidades da família no contexto domiciliar e planejar o cuidado a partir da valorização desta como corresponsável no processo saúde-doença. Necessita-se, portanto, conhecer a realidade, identificar as necessidades e, assim, orientar e estabelecer estratégias que facilitem o aprendizado e o desenvolvimento do cuidado pactuado à criança.

Diante do exposto, tem-se como objetivo do estudo analisar as publicações sobre oncologia pediátrica e as implicações para o cuidado de enfermagem.

\section{METODOLOGIA}

Trata-se de uma pesquisa bibliográfica do tipo narrativa de caráter descritivo-exploratório, com abordagem qualitativa, desenvolvida com base em material já publicado e constituído por artigos científicos.

De acordo com Gil (2010), a revisão bibliográfica é de extrema relevância, pois fornece fundamentação teórica ao trabalho bem como dá suporte e identifica o estágio atual do conhecimento referente à temática pesquisada, além de ter como principal vantagem o fato de permitir ao pesquisador a cobertura de uma gama de fenômenos amplos acerca do assunto investigado.

A busca foi realizada em fevereiro de 2016 na base de dados Literatura Latino-Americana e do Caribe em Ciências da Saúde (Lilacs). Utilizaram-se os seguintes descritores: "Enfermagem Pediátrica" e "Enfermagem Oncológica", previamente testados no Descritores em Ciências da Saúde (Decs). Destaca-se que não foi realizado recorte temporal, posto que se objetivou conhecer todas as produções existentes, independente da data de publicação.

Localizou-se 25 produções e estabeleceu-se como critérios de inclusão as publicações latino-americanas existentes na base de dados Lilacs, artigos completos, gratuitamente disponíveis na íntegra, publicados na língua portuguesa, inglesa ou espanhola. Como critérios de exclusão, caracterizaram-se as publicações que não eram artigos ou pesquisas, como teses, dissertações, reflexões e relatos de experiência, e produções com resumo incompleto e que não atendiam à temática proposta.

Sendo assim, foram selecionados para compor a amostra 13 artigos acerca da temática pesquisada. Após a análise dos resumos, realizou-se a inserção destes no quadro de extração de dados, composto por: código de 
identificação, objetivo, método, tipo de pesquisa, instrumento e análise, cenário/sujeitos, resultados, conclusão e código da amostra.

Nessa perspectiva, a avaliação dos dados obtidos baseou-se nas três etapas da análise de conteúdo temática propostas por Minayo, entre elas a pré-análise, composta pela leitura flutuante, constituição do corpus, formulação de hipóteses e objetivos; a exploração do material, que consiste essencialmente na operação de codificação e o tratamento dos resultados obtidos e interpretação (MINAYO, 2014).

Por conseguinte, a partir da análise de dados, emergiram três categorias: a ótica do cuidar da criança oncológica no contexto da família e da equipe de enfermagem; a repercussão dos sentimentos e emoções que permeiam o processo de adoecimento; e a promoção da qualidade de vida da criança oncológica e família.

\section{RESULTADOS E DISCUSSÕES}

Em relação à análise dos 13 estudos selecionados, eram pesquisas de campo, dentre estas, duas de caráter quantitativo e 11 qualitativo. No que respeita aos domínios, constatou-se que oito dos artigos reportaram-se a compreensão do cuidar da criança com câncer; dez, abordaram os sentimentos e emoções vivenciados; e cinco apresentaram aspectos que proporcionam a qualidade de vida, sendo que alguns destes eixos temáticos apareceram simultaneamente em mais de uma publicação. Diante do exposto, as três categorias são descritas a seguir.

\section{A ótica do cuidar da criança oncológica no contexto da família e da equipe de enfermagem}

Evidenciou-se neste estudo que seis artigos abordaram a compreensão de cuidado da criança para os familiares, permeado pela dedicação, abnegação, resiliência e obrigação moral. Para a família, as concepções do cuidar são como uma dádiva. Assim, em um movimento existencial mediado pela empatia dos profissionais de saúde com os cuidadores, é possível estabelecer um cuidado singular, integral e humanizado.

Neste sentido, Sales et al. (2015), destacam a importância da presença e participação constante dos pais no cuidado ao infante com câncer, visto que a família pode ser uma aliada na manutenção de sentimentos positivos e na melhoria da qualidade da assistência. Já Medeiros et al. (2014), consideram a família como sendo um sistema cujos membros estão relacionados, independente de laços consanguíneos, onde existe compromisso e vínculo e as funções de cuidado consistem na proteção, alimentação e socialização

A partir da análise dos estudos, identificou-se nos resultados a importância da assistência de enfermagem no cuidado à criança oncológica e família, enfatizando-se a ótica do cuidar na perspectiva da otimização e humanização do atendimento, bem como a utilização de estratégias que fortaleçam o vínculo de confiança entre eles. Diante deste contexto, a comunicação, acolhimento e apoio configuram-se como ferramentas eficazes para amenizar as fragilidades envolvidas no processo.

Desse modo, ao considerar a subjetividade dos indivíduos na ocasião do cuidado, deve haver um movimento de reflexão crítica das relações entre paciente, família e equipe para além da ênfase na integralidade da atenção. Trata-se de um processo que exige um destaque amplo e coletivo, não apenas do ser cuidado, prioridade da relação, mas também da preocupação com a estrutura do ambiente e da equipe multiprofissional, a fim de que todos usufruam de segurança e conforto (SANTOS et al., 2013).

De acordo com Fonseca (2013), o fato de incluir a família no cuidado à criança implica necessidade de a equipe de enfermagem estar aberta e atenta às interações, além de conhecer a dinâmica familiar, as formas de 
adaptação e o impacto das vivências. Somente, porém, essa perspectiva não basta, é preciso uma interação de saberes e responsabilidades compartilhadas para fomentar uma filosofia acerca do cuidar, com o intuito de reduzir incertezas sobre a efetividade do tratamento e ainda ofertar uma assistência perspicaz, pautada na escuta e no diálogo.

No que diz respeito ao profissional que atua em oncologia pediátrica, estudos abordam que o mesmo expressa que tem de separar o profissional do emocional no cotidiano de cuidado à criança que tem câncer, e descrevem a sua rotina diária dentro e fora do hospital, como também apontam a necessidade do planejamento e estabelecimento de estratégias voltadas à equipe que cuida, ao reconhecer que esta também precisa ser cuidada (MUTTI; PADOIN; DE PAULA, 2012).

Nessa perspectiva, é imprescindível a valorização dos profissionais de enfermagem como sujeitos protagonistas no cenário de atuação, seja na atenção primária ou no ambiente hospitalar, pois também estão propensos ao risco de adoecimento em razão de conviverem rotineiramente com situações de vulnerabilidade, que são desencadeadas pelo sofrimento e fragilidade das crianças acometidas por neoplasias.

\section{A repercussão dos sentimentos e emoções que permeiam o processo de adoecimento da criança}

Retrata-se, pela presente pesquisa, que a revelação do diagnóstico de câncer infantil ocasiona uma desestruturação familiar, uma vez que se gera uma grande expectativa em pessoas de seu entorno pela alteração de sua dinâmica. Além disso, pode produzir sensações de impotência e desesperança quanto ao prognóstico caracterizado por questões culturais relacionadas à doença. Os estudos mostram que todas essas reações, tanto da família quanto da própria criança, criam situações conflitantes em seus mundos, pois ambos têm suas atividades de vida diária alteradas.

Pelo ambiente desagradável propiciado pelo hospital à criança enferma e sua família, destaca-se alguns sentimentos envolvidos nas recorrentes internações hospitalares, como o estresse e a angústia, que repercutem na vivência de situações de extrema fragilidade física e psicossocial. Assim, a enfermagem exerce um papel crucial no atendimento ao binômio criança/família, uma vez que as atitudes de cuidado devem estar voltadas à minimização desses fatores negativos e promoção de uma assistência humanizada que fortaleça laços de confiança e amizade e mostre que ambos são coparticipantes do processo (SANTOS et al., 2013).

Malagutti (2011) acredita que a criança, ao deixar temporariamente de realizar as atividades que fazem parte do seu cotidiano, como ir à escola, brincar e conviver com seus amigos, e quando seu universo passar a ser o tratamento, as rotinas de consultas e internações hospitalares, tem uma sobrecarga da doença, não somente para ela, mas também para seus familiares e amigos. Além disso, o distanciamento do ambiente familiar, a realização de procedimentos dolorosos e a aproximação de pessoas estranhas, constantemente causam o aumento de fatores estressores na vida desses indivíduos.

Nesse contexto, cabe apontar a relevância do papel da equipe de enfermagem, que pode e deve proporcionar uma assistência integral. Ou seja, valorizar as questões biológicas, psicológicas, sociais, econômicas, espirituais e culturais da criança e de seus cuidadores, com o intuito de minimizar o sofrimento deste processo, que envolve um misto de emoções como estresse, medo, angústia, insegurança, incerteza, enfim, situações desgastantes para ambos (FONSECA, 2013).

Ao cuidar do binômio criança e família, a equipe necessita estar voltada para a prevenção de agravos, promoção do conforto, bem-estar e saúde, como também atentar para o manejo da dor e alívio dos sinais e sintomas (MALAGUTTI, 2011). Além disso, ações de apoio, valorização, respeito e atendimento às necessidades 
humanas básicas precisam estar fortalecidas na prática dos profissionais no intuito de minimizar sentimentos negativos desses usuários.

\section{A promoção da qualidade de vida da criança oncológica e sua família}

Denota-se, com os resultados encontrados no estudo, que a qualidade de vida contribui significativamente para a resposta tanto da adesão ao tratamento quanto da possibilidade de enfrentamento da doença de diversas formas. Com isso, a criança e a família permanecem em um ambiente mais harmonioso, posto que a equipe de enfermagem preocupa-se em promover o bem-estar e uma relação humanística com o binômio supracitado.

Nesse contexto, um dos resultados da pesquisa mostra que o uso do Cateter Venoso Central de Inserção Periférica (PICC) como um dos dispositivos usados na terapia venosa de quimioterápicos, o qual se constitui em uma opção confiável, pois permite uma assistência adequada, mesmo que seja por períodos que não atendam a todo o tratamento, contribui positivamente para a qualidade de vida das crianças com câncer.

Ao discorrer sobre o uso do PICC, Petry et al. (2012) destacam a necessidade de aperfeiçoar os conhecimentos de enfermagem no sentido de qualificar a assistência, e isso faz com que se busque e se incorpore cada vez mais novas tecnologias de cuidado. Logo, esse dispositivo veio somar, sendo inserido à assistência de enfermagem a fim de contribuir na melhoria da qualidade de vida do paciente pediátrico.

No âmbito do ambulatório, outro estudo descreve o uso do brinquedo terapêutico como forma de preparar as crianças para a realização de tratamentos, como a quimioterapia. Este instrumento de cuidado apresentou grande valor ao favorecer a compreensão do infante e facilita a interação deste com o profissional, além de tornar a permanência no ambulatório agradável e descontraída.

O brinquedo terapêutico mostra-se uma forma de comunicação com a criança, pois proporciona diversão, relaxamento, diminuição da ansiedade e alívio das tensões. Trata-se de um meio de expressar os sentimentos, ter uma recuperação mais efetiva, além de melhor aceitação ao tratamento. O lúdico ainda cria condições de a criança entender e aceitar melhor o que ocorre com ela, e ainda contribui para uma maior tranquilidade e segurança no que se refere ao processo de adoecimento (FONTES et al., 2010).

Em contrapartida, uma das produções aponta os efeitos colaterais relatados pelas crianças em decorrência da quimioterapia, dentre os quais estão as náuseas e vômitos, aumento de peso, reação de hipersensibilidade, fadiga e febre. Estes interferem diretamente no cotidiano delas, levando a uma diminuição do estado de bemestar e, consequentemente, piora na qualidade de vida.

Segundo Cicogna, Nascimento e Lima (2010), o tratamento quimioterápico promove uma série de transformações na vida daqueles que o recebem, pois altera seu corpo, estado emocional e rotina, bem como de seus familiares. Nessa trajetória, as crianças convivem com sentimento de tristeza, medo, ansiedade e depressão, todavia, apesar dos efeitos colaterais, a quimioterapia ainda é encarada como fonte de vida.

Sendo assim, os profissionais de saúde necessitam considerar a visão do enfermo acerca da doença e entender essa experiência sob a sua ótica. Busca-se como fonte de informação a própria criança, tendo em vista que ela é a principal conhecedora de suas necessidades relacionadas com o bem-estar e valores humanos, no intuito de ajudá-la a buscar novos modos de viver (CICOGNA; NASCIMENTO; LIMA, 2010).

Outra forma apontada como promoção da qualidade de vida são os cuidados paliativos, que se configuram como elementos eficazes no cuidado à criança com câncer, especialmente quando a assistência está alicerçada 
na Teoria Humanística de Enfermagem, que elucida o atendimento com um diálogo entre os seres humanos, os quais estão dispostos a integrar um relacionamento existencial um com o outro (SÁ FRANÇA et al., 2013). Esta interação proporcionada pela comunicação fortalece o apoio mútuo entre equipe, família e criança.

Nesta perspectiva, Malagutti (2011) informa que os cuidados paliativos ofertados pela equipe de saúde à criança são uma forma de abordagem holística, centrada na maximização da sua qualidade de vida, bem como do alívio da dor e dos sintomas da criança no intuito de proporcionar conforto a ela e sua família, além de atender as necessidades biopsicossociais e espirituais e de apoio.

\section{CONSIDERAÇÕES FINAIS}

A realização deste estudo oportunizou desvelar e agregar importantes subsídios da enfermagem pediátrica e oncológica, bem como conhecer questões acerca das implicações de cuidar desta criança para a família e equipe de saúde, tendo em vista que a oncologia se constitui em uma área extremamente delicada e estigmatizada pela sociedade.

Faz-se necessário, no entanto, que o trabalho em equipe seja multidisciplinar, necessidade esta imposta pelo progresso da globalização para fomentar uma filosofia de vida e cuidado a fim de que o respeito esteja sempre presente do primeiro ao último atendimento, com o intuito de transcender os paradigmas vigentes do cuidado e promover uma assistência singular, integral e otimizada, embasada nos preceitos éticos e morais que alicerçam a profissão da enfermagem.

Nesse sentido, conclui-se que a equipe de enfermagem não deve se limitar apenas aos protocolos terapêuticos, mas também incluir ações humanizadas que valorizem a participação constante da família nesse contexto, além do suporte social e psicológico ofertado pelos pais a esta criança, que são imprescindíveis para o enfrentamento e adesão ao tratamento, posto que a cura não deve ser baseada apenas no restabelecimento biológico, mas no bem-estar e qualidade de vida dessas crianças.

\section{REFERÊNCIAS}

AVANCI, B. S. et al. Cuidados paliativos à criança oncológica na situação do viver/morrer: a ótica do cuidar em enfermagem. Escola Anna Nery Revista Enfermagem, Rio de Janeiro, v. 13, n. 4, out./dez. 2009.

BRASIL. Ministério da Saúde, 2010. Estatuto da Criança e do Adolescente. Disponível em: 〈http://www.assistenciasocial.al.gov.br>. Acesso em: 12 out. 2015.

- Ministério da Saúde. Instituto Nacional do Câncer. Estimativas do câncer infantil. Brasília, 2014. Disponível em: <http://www.inca.gov.br>. Acesso em: 5 out. 2015.

- Ministério da Saúde. Instituto Nacional do Câncer. Particularidades do câncer infantil. Brasília, 2008. Disponível em: <http://www.inca.gov.br>. Acesso em: 5 out. 2015].

Ministério da Saúde. Instituto Nacional do Câncer. Tipos de câncer infantil. Brasília, 2011. Disponível em: <http://www2.inca.gov.br>. Acesso em: 20 set. 2015.

CICOGNA E.C.; NASCIMENTO L.C.; LIMA R.A.G. Crianças e adolescentes com câncer: experiências com a quimioterapia. Rev. Latino-Americana Enfermagem, São Paulo, 18(5):[09 telas], set./out. 2010.

FONSECA, Ariadne S. Enfermagem pediátrica. 1. ed. São Paulo: Martinari, 2013. 
FONTES, C. M. B. et al. Utilização do brinquedo terapêutico na assistência à criança hospitalizada. Revista Brasileira de Educação Especial, Marília, v. 16, n. 1, p. 95-106, jan./abr. 2010.

GOMES, I. P.; COLLET, N.; REIS, P. E. D. Ambulatório de quimioterapia pediátrica: a experiência do aquário carioca. Texto \& Contexto - Enfermagem, Florianópolis, v. 20, n. 3, jul./set. 2011.

GIL, Antônio Carlos. Como elaborar projetos de pesquisa. 5. ed. São Paulo: Atlas, 2010.

MALAGUTTI, Willian. Oncologia pediátrica: uma abordagem multiprofissional. 1. ed. São Paulo: Martinari, 2011.

MEDEIROS G. M. S. et al. Repercussões do câncer infantil no cotidiano do familiar cuidador. Revista da Rede de Enfermagem do Nordeste, v. 15, n. 2, p. 233-239, mar./abr. 2014.

MINAYO, Maria Cecília S. O desafio do conhecimento: pesquisa qualitativa em saúde. 14. ed. São Paulo: Hucitec, 2014.

MUTTI, C. F.; PADOIN, S. T. M.; DE PAULA, C. C. Espacialidade do ser-profissional-de-enfermagem no mundo do cuidado à criança que tem câncer. Escola Anna Nery Revista Enfermagem, Rio de Janeiro, v. 16, n. 3, jul./set. 2012.

PETRY, J. et al. Cateter venoso central de inserção periférica: limites e possibilidades. Revista Eletrônica de Enfermagem, Goiânia, v. 14, n. 4, p. 937-943, out./dez. 2012.

SÁ FRANÇA J. R. F. et al. Importância da comunicação nos cuidados paliativos em oncologia pediátrica: enfoque na teoria humanística de enfermagem. Rev. Latino-Americana Enfermagem, São Paulo, v. 21, n. 3, p. Tela 1-Tela 7, maio/jun. 2013.

SALES, C. A. et al. Presença familiar no olhar existencial da pessoa com câncer: compreendendo o fenômeno à luz heideggeriana. Revista Eletrônica de Enfermagem, Goiânia, v. 17, n. 1, p. 30-36, jan./mar. 2015.

SANTOS, M. R. et al. Desvelando o cuidado humanizado: percepções de enfermeiros em oncologia pediátrica. Texto \& Contexto Enfermagem, Florianópolis, v. 22, n. 3, jul./set. 2013.

SILVA, T. P. da et al. Cuidados de enfermagem à criança com câncer: uma revisão integrativa da Literatura. Revista de Enfermagem da UFSM, Santa Maria, v. 3, n. 1, p. 68-78, jan./abr. 2013. 